\title{
New methods, measures, and models for analyzing memory impairment using triadic comparisons
}

\author{
Michael D. Lee ${ }^{1} \cdot$ Melinea Abramyan $^{1}$ - William R. Shankle ${ }^{2}$
}

Published online: 28 October 2015

(C) Psychonomic Society, Inc. 2015

\begin{abstract}
We study the effect of memory impairment on triadic comparisons of animal names in a large clinical data set. We define eight groups of subjects in terms of their delayed free recall performance, and present standard analyses of the triadic comparison and free recall data that provide little insight into the effect of memory impairment on semantic structure. We then develop and apply two new methods for analyzing the data, based on cognitive models and using Bayesian statistical inference. The first new method focuses on modeling changes in semantic representation, by inferring multidimensional scaling (MDS) representations for each group based on their triadic comparisons. These representations reveal a successive decrease in semantic cluster structure and increase in uncertainty with increasing impairment. We propose a measure of spatial organization as a means of quantifying the visually evident changes in semantic organization, and demonstrate its usefulness. The second new method focuses on modeling changes in memory access with impairment, inferring the extent to which each individual makes triadic comparisons consistent with a common semantic representation. Although these inferences are based on just 12 comparisons per subject, we show that they vary systematically with memory impairment group. We conclude by discussing the potential for clinical application of our new models, measures, and methods.
\end{abstract}

Michael D. Lee

mdlee@uci.edu

1 Department of Cognitive Sciences, University of California, Irvine, CA, USA

2 Medical Care Corporation, Newport Beach, CA, USA
Keywords Triadic comparison · Multidimensional scaling $\cdot$ Alzheimer's disease and related disorders

\section{Introduction}

One of the most basic roles of memory is to structure and provide access to information so that it captures and conveys meaning. Semantic structure enables the fundamental cognitive capabilities of generalization and prediction, and allows what people have learned and experienced to guide their current understanding and behavior. For these reasons, one of the most serious potential impacts of memory impairment is the degradation of semantic representation, or degradation of the access people have to their representation. Failing to understand the meaningful relationships between stimuli severely impacts a person's functional abilities.

There is compelling evidence that Alzheimer's disease and related disorders (ADRD) are associated with the disruption of semantic knowledge. One source of evidence comes from fluency tasks, including categorical fluency tasks in which subjects are asked to generate exemplars of a specific category (e.g., furniture), and phonemic fluency tasks asking subjects to generate words that start with a specific letter. The standard finding is that $\mathrm{AD}$ subjects generate considerably fewer exemplars in these sorts of fluency tasks than do healthy controls (e.g., Fagundo et al., 2008)

Another source of evidence comes from studying semantic structures, examining the relationships people have between the concepts represented by words. A central task in this approach is triadic comparison, in which three stimuli are presented on each trial, and the person is asked to identify the "odd one out". For example, a trial might involve the presentation of the animal names "goat", "deer", and "rat", and the person might choose "rat" as the odd one out. 
Romney et al. (1993) used the decisions people made in a triadic comparison task to measure the similarity between pairs of animal name stimuli, and then developed multidimensional scaling representations (MDS: Shepard, 1974, 1980) of the semantic structure. In MDS representations, stimuli are represented by points in a multidimensional space in which the distance between points represents the similarity of each pair of stimuli, such that more similar stimuli are located closer to each other. Romney et al. (1993) showed that MDS representations predicted wellestablished effects of semantic clustering in the order words are generated in free recall (Bousfield, 1953).

The use of triadic comparisons has been applied and extended by a number of authors to study changes and differences in semantic structure related to ADRD and other clinical conditions, including schizophrenia and frontal lobe damage (e.g., Au et al. 2003; Sylvester \& Shimamura, 2002; Tallent et al., 2001). MDS representations have often been a focus of this work, but other representational formalisms from the similarity-modeling literature-including extensions of MDS to include individual differences like INDSCAL (Arabie et al., 1987), and other forms of representation, such as those based on trees like ADDTREE (Corter, 1996) or more general networks like PATHFINDER (Schvaneveldt et al., 1989), as well as those based on various forms of cluster analysis (Gordon, 1999) — have also been used. For example, Chan et al. (1995) investigated the semantic structure of animals in normal controls and subjects with Huntington's disease and ADRD using INDSCAL and PATHFINDER representations. They found that the representations of ADRD subjects had weaker associations between closely related animals, lacked the clustered organization following major features like size and domesticity, and generally showed a breakdown in semantic structure. Chan et al. (2001) extended this line of research to consider different semantic domains, finding differences in the effect of ADRD on the domains of animals and tools.

Another way to understand the effect of memory impairment is in terms of access to semantic structures. For example, Elvevåg and Storms (2003) cautioned, based on an observed lack of inter-individual consistency, against the assumption that schizophrenic subjects have different semantic representations in the context of MDS representations and triadic comparison data. Elvevåg et al. (2005) present further evidence that subjects with schizophrenia do not have different semantic structures from normal controls, for animal and food stimulus domains. The alternative explanation for schizophrenics explored by Elvevåg et al. (2002) involves differences in access to semantic structures, and this theoretical perspective is advocated in the context of $\mathrm{AD}$ subjects and MDS representations by Storms et al. (2003). Voorspoels et al. (2014) presented a recent summary of this theoretical perspective, raising the possibility that understanding impairment might not be best achieved by focusing on representational change, but rather on changes in access to representations. The use of cognitive models is central to both approaches. Changes in semantic structure are only implicit in the pattern of triadic comparison choices, and require the use of a representational model to be revealed in an interpretable way. Similarly, if impairment is conceived as affecting access to a semantic structure, a cognitive model of both the structure and the task-relevant decision-making process is required.

Against this background, the goal of this paper is to extend the set of representational models, measures, and methods available to undertake research on clinical changes in triadic comparison data. We develop and demonstrate these new models and methods using a new and extensive clinical data set, involving both a triadic comparison task and delayed free recall task for animal name stimuli. Our contribution to methods involves the development of a Bayesian approach, based on graphical models and computational sampling methods, for inferring MDS representations from individual-level trial-by-trial triadic comparison data. The Bayesian approach has the advantage of characterizing the uncertainty of inferences about semantic representations. Our contribution to measures involves the application of a measure of spatial randomness as a simple statistical summary of the cluster structure in MDS representations. Our contribution to models involves the development of a new individual differences model that provides an alternative to existing models like INDSCAL. Collectively, our models span a range of theoretical possibilities, ranging from the effect of memory impairment being on underlying semantic structures, to the effect being in differences in access to a common and stable semantic structure.

The remainder of this paper is organized as follows. We first describe the clinical data set, and our use of performance on a delayed free recall task to divide the subjects into 8 groups with different levels of impairment. We then report some basic analyses of the behavioral data, to contrast with the modeling analyses that are the main focus of the paper. The basic data analyses examine how often each animal is chosen as the odd one out, how often it is not recalled, and the distribution of similarities between animals implied by the triadic comparison choices. We argue that these analyses are not very insightful, and that modeling approaches are warranted. Our first modeling analysis develops and applies a Bayesian approach to MDS applicable at the group level, and we observe large changes in the semantic organization revealed by these representations for different groups. We then develop a measure of the spatial randomness of the MDS representations that quantifies this change in semantic structure. Our second modeling analysis also involves inferring an MDS representation, but assumes a common semantic representation, allowing for individual differences 
in access through differences in response determinism. The results show a relationship between these individual differences and the severity of impairment at the individual level. We conclude with a discussion of the potential applications of our new measures and models, and a discussion of possible future developments.

\section{Methods and data}

Our data set consists of 2,922 subjects from a cognitive disorders clinic, a primary care clinic, and a long-term care insurance application pool. Subjects from the clinical sample ranged in severity from normal cognition to moderately severe dementia, and were assessed one or more times as part of their longitudinal care, such that there were a total of 4,834 assessments available for analysis. Subjects from the cognitive disorders clinic were diagnosed for the underlying etiology of their dementing disorder by the same clinical neurologist using standardized diagnostic criteria for AD, Lewy Body Disease, Cerebrovascular Disease, Normal Pressure Hydrocephalus, and Frontal Temporal Lobe Disease. The cognitive testing consisted of the MCI Screen of memory, language and executive function, orientation to date, the CERAD Drawings constructional praxis task, the Trails A and B sequencing and set-shifting tasks, the Ishihara Color Plates number naming object recognition task, and the F-A-S phonemic fluency task. Of the 2,922 subjects assessed, the triadic comparisons task was successfully completed for 4,663 assessments of 2,712 subjects.

From this data set, we consider just the first visit of each of the 2,712 individual subjects, and only their performance on tasks relating to the animals. The two relevant tasks are the triadic comparisons of the animals, and the subsequent free recall of the animals, which are both part of the MCI Screen. The data set uses a total of 21 animals, a subset of nine of which were chosen at random for each subject. The subject completed a total of 12 triadic comparisons for these nine animals, following a standard lambda-1 design (Burton \& Nerlove, 1976), which balances the pairings of the animals across the triads, so that each animal is presented in four triads. In the delayed free recall task, the subject simply had to recall as many of the nine animals as possible.

\section{Defining severity of impairment groups}

Since our goal is to understand the effects of memory impairment on semantic judgments, we used free recall performance as a basis for determining different impairment groups, reserving the triadic comparison to study semantic memory. The basic assumption is that subjects with greater impairment will perform worse on the free recall task, both in terms of failing to recall and generating intrusions. Other criteria for defining groups of impaired subjects are obviously possible-such as, for example, an independent clinical assessment like a FAST staging (Reisberg, 1988) — and some may be more desirable. Our use of free recall performance is based on the availability of those data, the lack of availability of other possibly useful clinical data, and the fact that the free recall task used the animal words also used in the triadic comparison task.

It is important to note that free recall behavior is used solely for measuring impairment and defining groups, and not in assessing the performance of those groups, while triadic comparison performance is used solely to assess semantic judgments, and not to define the groups. Thus, while both the free recall tasks and triadic comparison task are presumably affected by the memory impairment of subjects, there is nothing circular in our approach. On the contrary, our approach is naturally interpreted as a "common cause" hierarchical model, in which a latent psychological structure is manifest in multiple behavioral phenomena (Lee, 2011).

Following this analysis strategy, Fig. 1 summarizes subject performance on the delayed free recall task, and shows the exact criteria by which we grouped subjects for subsequent analyses. The main panel shows by the area of circles and labeled counts the joint distribution of the number of animals correctly recalled, between 0 and 9 , and the number of intrusions recalled, between 0 and the observed maximum of 6 . The marginal distributions of these measures are shown by bars below and to the left.

The 8 different groups of subjects we constructed from these performance measures are shown by the rectangles with dashed line borders. The 331 subjects who recalled all nine animals, with no intrusions, are placed in group I. The $427+69=496$ subjects who recalled eight of the nine animals, with at most one intrusion, are placed in group II, and so on. In total there are eight groups, defined by progressively worse performance in terms of correct recall and the presence of intrusions. The exact bounds shown by the rectangles in Fig. 1-and, in particular, the larger range used for group VII-were chosen to try and insure comparable numbers in each of the eight groups.

Of course, there are other ways in which the free recall performance could be used to define groups of subjects. There is nothing special, for example, about the choice of eight groups. It would be possible to consider different classification patterns that treated failures to recall and intrusions differently, perhaps based on theorizing about how different levels of caution in responding might differentially affect these two types of errors. The method of partitioning we used-made explicit in Fig. 1-is one 


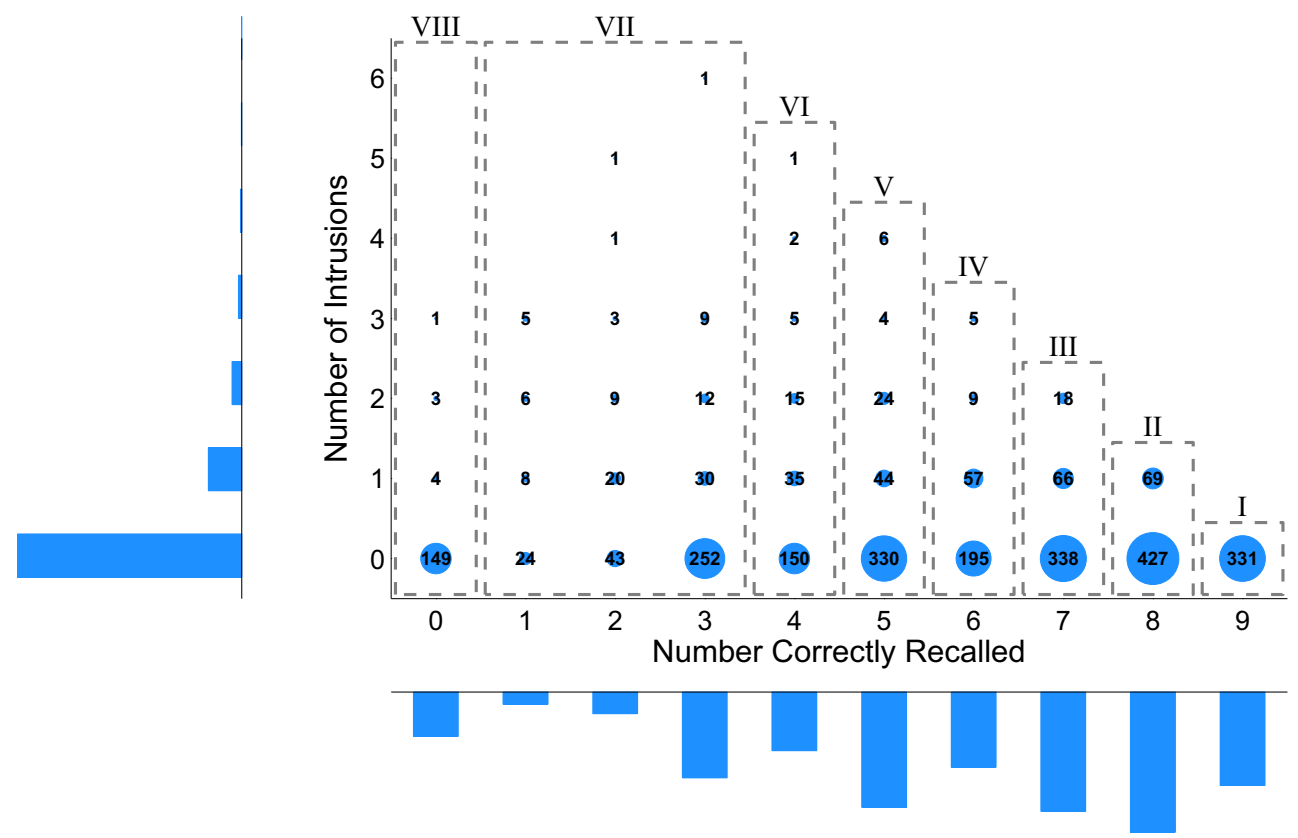

Fig. 1 The joint distribution of correct recall and intrusions, and the classification of subjects into eight groups based on these measures of performance in the free recall of animals task

simple and reasonable approach, and allows us to demonstrate the new methods and measures that are our intended contribution.

\section{Basic behavioral analyses}

Our goal is to understand the changes in semantic memory evident in the triadic comparisons and free recall answers across the eight groups, with their different levels of impairment. In this section, we consider a number of simple analyses based directly on the basic behavioral data. These behavioral analyses provide a context for assessing the usefulness of the subsequently developed model-based analyses.

\section{Odd one out choices}

Figure 2 summarizes the odd one out choices for each of the eight groups. Each panel corresponds to a group. The first panel, corresponding to group I, shows the proportion of times, over all subjects in that group, and all triads including the animal, that each animal was chosen as the odd one out. It shows, for example, that for subjects in group I, with perfect recall performance, "rat" was most frequently chosen as the odd one out, while "zebra" was the least frequently chosen. The remaining panels in Fig. 2 show the difference in the proportion of times each animal was chosen by all of the subjects in that group, when compared to the group I proportions. Thus, for example, subjects in group
VIII chose "antelope" and "zebra" as the odd one out choice more frequently than subjects in group I.

There is at least one clear conclusion that can be drawn from the odd one out analysis in Fig. 2. In general, the absolute level of difference in proportions from group I grows as impairment progresses from group II through to group VIII. Specific meaningful change at the level of the animals is more difficult to discern. The frequency with which "antelope" and "zebra" are chosen generally increases across groups, but there are few other obvious regularities. Similarly, the proportions present for group I do not seem have any natural interpretation in terms of obvious properties like the frequency of the animal word, the size of the animal, its status as a wild or domestic animal, or other salient features.

\section{Recall failures}

Figure 3 summarizes the ability of subjects in the different groups to recall each of the animals during the free recall task. By definition, there are no failures for group I, but the remaining panels show the proportion of times, across all subjects and all animals that were not recalled, that the failure occurred for each animal. For group VIII this analysis simply reduces to the proportion of times each animal was presented, since no animals were correctly recalled by subjects in this group. For groups II-VII, however, the proportions carry meaningful information, showing differences in recall proportions. 

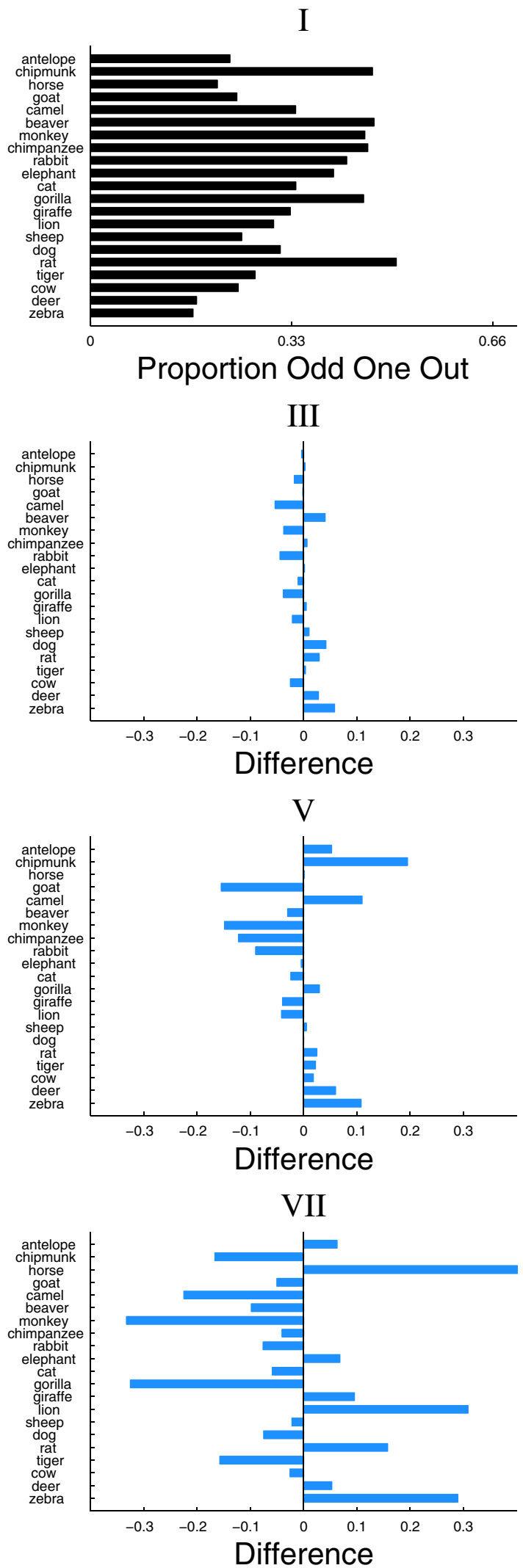

Fig. 2 Odd one out choices for each of the 21 animals for all eight groups. The top-left panel for group I shows the proportion of times each animal was chosen as the odd one out for a triad in which it was
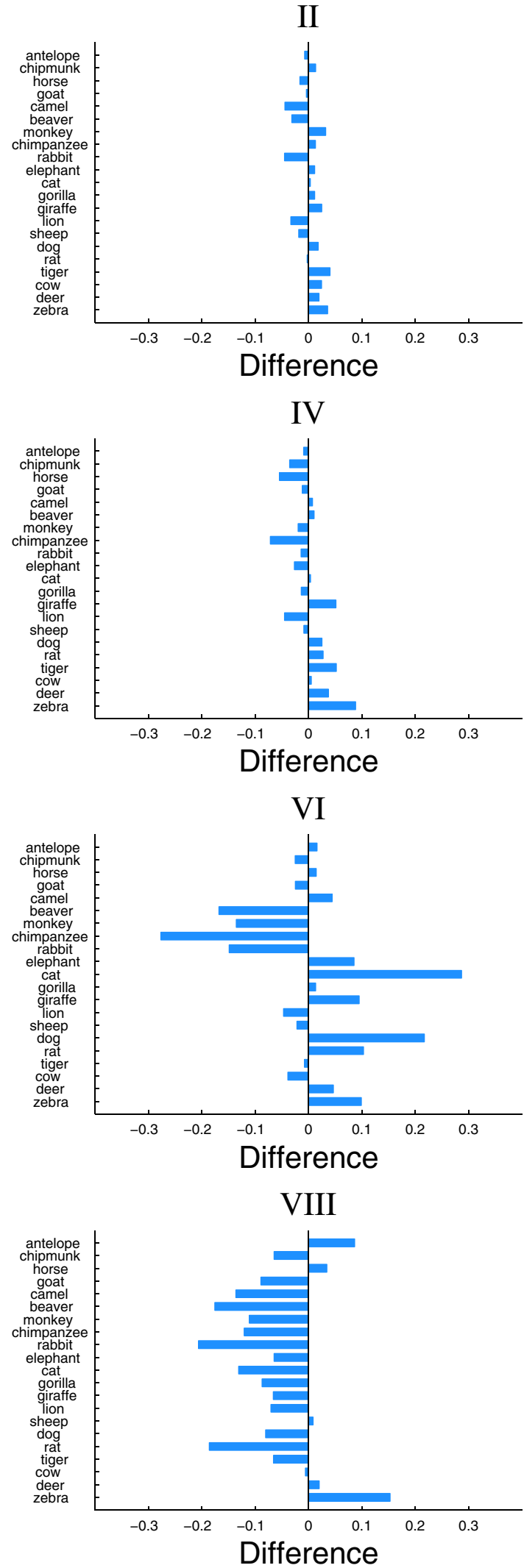

present. The remaining panels for groups II-VIII show the difference in this proportion for these groups when compared to group I as a reference 

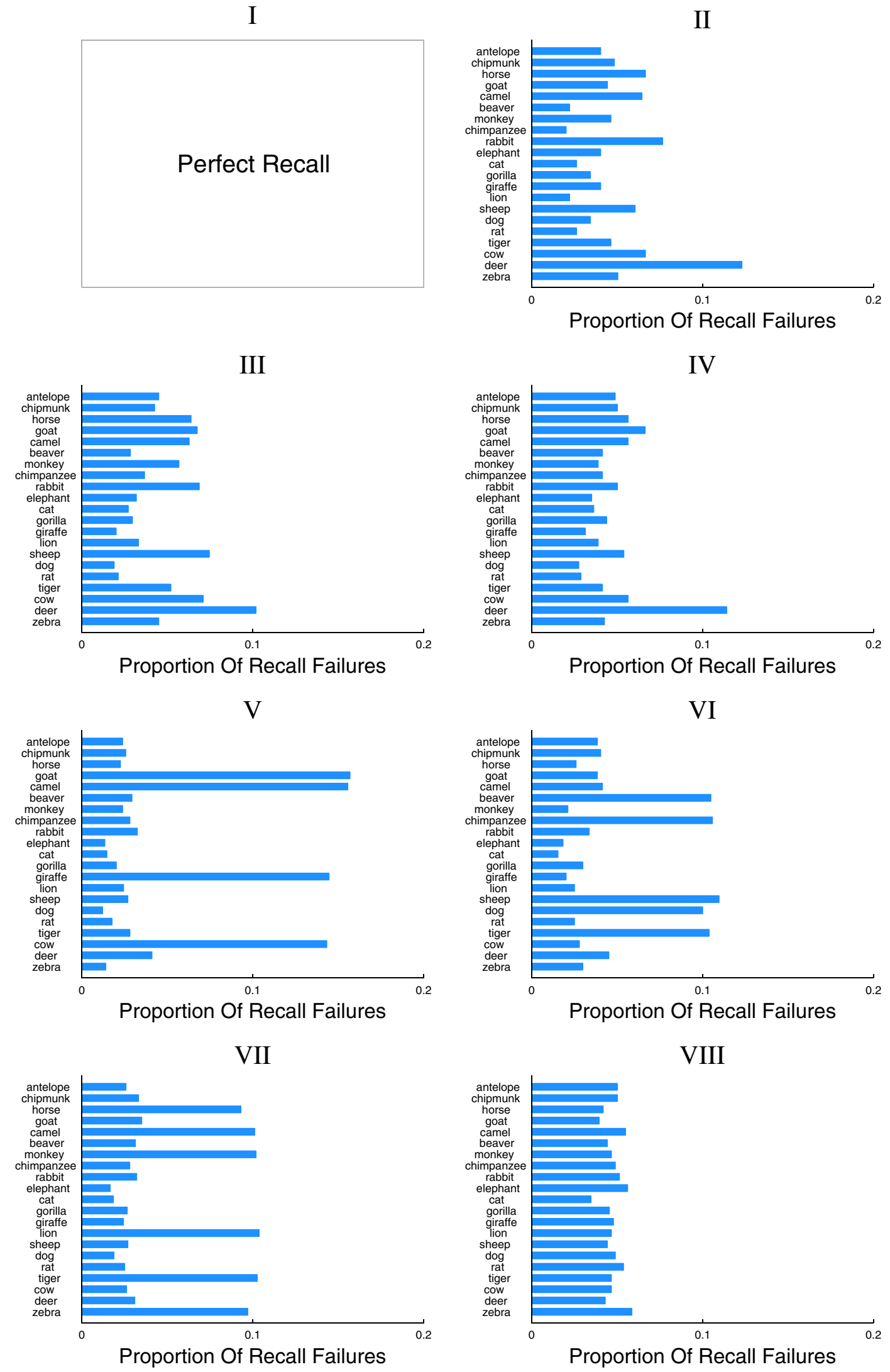

Fig. 3 The proportion of times, among all failures to recall, that each animal was not recalled in the delayed free recall task, for all subjects in groups I-VIII 
There are some interesting observations that can be made from Fig. 3. For many groups, "deer" is frequently not recalled, but "dog" and "cat" are rarely not recalled. There are no clear patterns, however, in terms of changes across successive groups. The level of failure to recall specific animals, or sets of animals, does not appear to be diagnostic of group membership.

\section{Pairwise similarities}

Figure 4 shows the results of an analysis measuring the similarity between pairs of animals based on the triadic comparisons. The similarity between each possible pair of animals is assumed to be the proportion of times, over all triads in which both animals were presented, that neither was chosen as the odd one out. The intuition is that the most different animal is chosen as the odd one out, and so the remaining pair of animals in the triad are more similar to each other. Figure 4 shows the distribution of these pairwise similarity values over all animal pairs, based on all of the choices made by the subjects in groups I-VIII.

The distributions in Fig. 4 change over the eight groups. Groups corresponding to less impaired subjects, for example, skew with longer tails towards higher similarity values, whereas groups corresponding to greater impairment are more symmetric. For some groups-such as groups V, VI, and VIII-there is some suggestion of multi-modality, with a set of very high pairwise similarities. It is difficult, however, to interpret any of these changes in the mea-

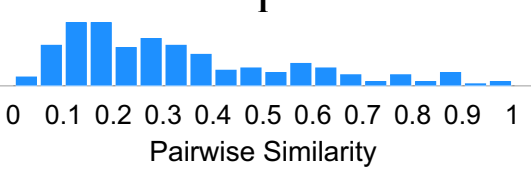

III

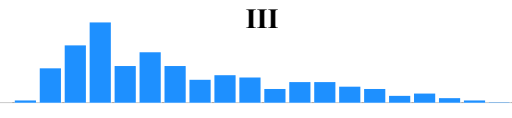

$\begin{array}{lllllllllll}0 & 0.1 & 0.2 & 0.3 & 0.4 & 0.5 & 0.6 & 0.7 & 0.8 & 0.9 & 1\end{array}$ Pairwise Similarity

V

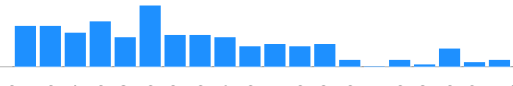

$\begin{array}{lllllllllll}0 & 0.1 & 0.2 & 0.3 & 0.4 & 0.5 & 0.6 & 0.7 & 0.8 & 0.9 & 1\end{array}$ Pairwise Similarity

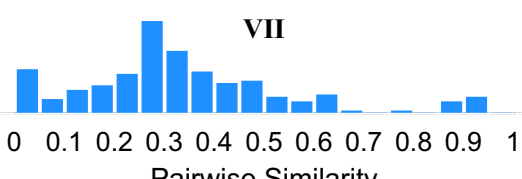

Pairwise Similarity sured pairwise similarities in terms of changes to semantic structure.

\section{Group multidimensional scaling model}

The analyses presented in the previous section considered basic behavioral measures of the triadic comparison and free recall tasks, as they varied by the eight groups defined to correspond to different levels of memory impairment. While these analyses afforded some limited findings, none provided great insight into the basic question of how the semantic representation of the animals changed with impairment.

In this section, we explore an alternative cognitive modeling approach to analyzing semantic representations inherent in the triadic comparison data. Our approach is based on an MDS model of semantic representation of the animal domain, and a novel Bayesian approach to inferring these representations from the triadic comparison choices. Thus, this model takes the theoretical perspective that the effects of impairment can be understood in terms of changes to semantic representations.

\section{Generative model}

We use graphical modeling to formalize our assumptions about the MDS representational model, and its relationship to the triadic comparison decisions. Graphical models are a convenient formalism for expressing and making

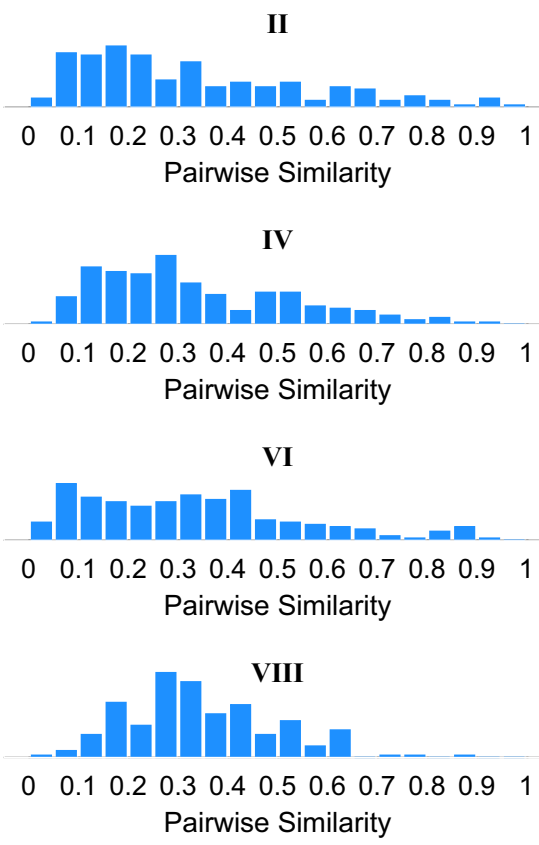

Fig. 4 The distribution of pairwise similarity values - measured for each pair of animals as the proportion of triads in which both were presented but neither chosen as the odd one out-for groups I-VIII 


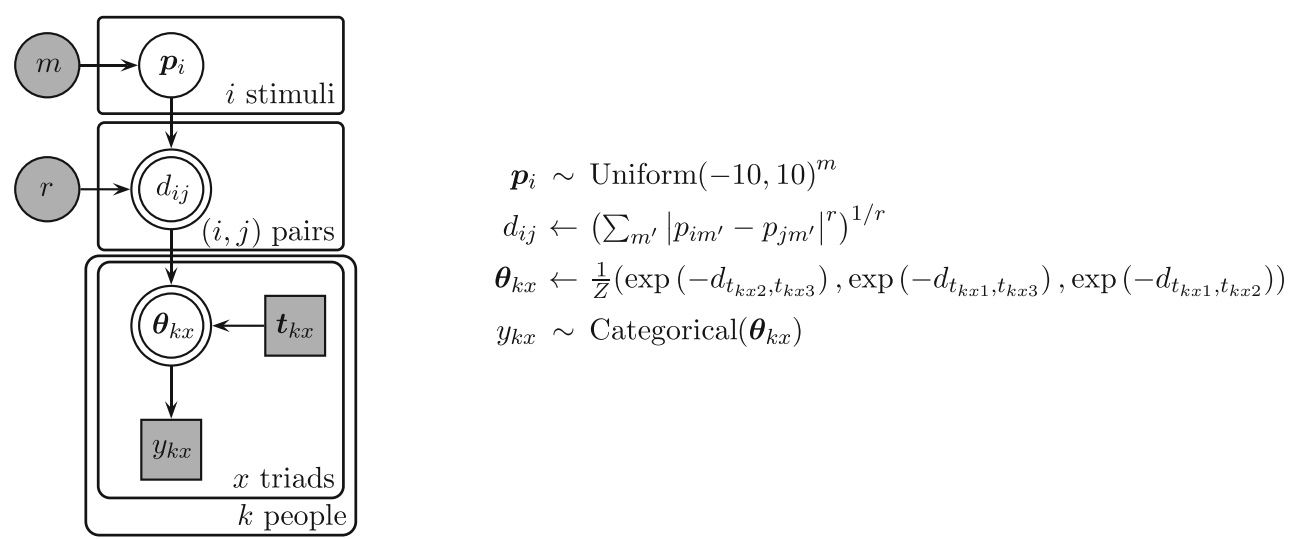

Fig. 5 Graphical model for inferring a spatial representation based on triadic comparisons

inferences with probabilistic cognitive models (Lee and Wagenmakers, 2013). They quantify the way in which latent model parameters generate observed behavioral data. Typically, unobserved parameters are shown by unshaded nodes, observed data shown by shaded nodes, and the graph structure indicates the dependencies between them. Other observed values, such as properties of the experimental design, are also shown as shaded nodes, and deterministic variables are shown as double-bordered nodes. Encompassing plates denote independent replications of the graph structure, typically iterating over people, trials, or stimuli.

Figure 5 shows the graphical model we use. It assumes that stimuli are represented as points in a multidimensional space, and the distances between them correspond to similarities that determine the choice probabilities in triadic comparisons. Thus, the latent coordinate locations $p_{i}=\left(p_{i 1}, \ldots, p_{i m}\right)$ representing the $i$ th stimulus in an $m$ dimensional space generate pairwise distances $d_{i j}$ between the $i$ th and $j$ th stimuli according to the Minkowski $r$-metric. The three stimuli presented on the $x$ th triad to the $k$ th person are $t_{k x}=\left(t_{k x 1}, t_{k x 2}, t_{k x 3}\right)$.

The key modeling assumption is that the probability of choosing each of these three stimuli as the odd one out depends on the three pairwise distances between these stimuli. In particular, the probability of choosing $t_{k x 1}$, represented by $\theta_{k x 1}$ is proportional to the similarity between the other two stimuli, modeled as an exponential decay of the distance between them given by $\exp \left(-d_{t_{k x 2}, t_{k x 3}}\right)$. The modeling choice of an exponential decay function relating stimulus similarity to distance in an MDS representation is given a theoretical basis by Shepard's (1987) "Universal Law of Generalization", and is widely used in cognitive process models of identification, categorization, and decisionmaking (see Nosofsky 1992, for an overview). The intuition behind using the similarity of the two stimuli not chosen is that a stimulus is more likely to be chosen as the odd one out if the other two are similar to each other. The final part of the generative model simply assumes that the chosen stimulus $y_{k x}$ is a categorical draw from the choice probabilities $\theta_{k x}=\frac{1}{Z}\left(\theta_{k x 1}, \theta_{k x 2}, \theta_{k x 3}\right)$ where $Z$ is a normalizing constant to insure the probabilities sum to one.

An important advantage of the graphical modeling approach is that it makes it practical to conduct fully Bayesian inference. We implemented the graphical model in JAGS (Plummer, 2003), which uses Markov-Chain MonteCarlo (MCMC) methods to return samples from the joint posterior distribution of the parameters conditional on the model and data. ${ }^{1}$ The joint posterior of the coordinate location parameters represents the uncertainty about the MDS representation of the stimuli, based on the model and behavioral data.

Probabilistic multidimensional scaling models have previously been developed in cognitive psychology, originally by making parametric assumptions about the posterior distribution (e.g., Zinnes \& Mac Kay, 1983), and more recently without these over-constraining assumptions using MCMC methods (e.g., Okada \& Shigemasu, 2009). As far as we are aware, the model in Fig. 5 is the first probabilistic MDS approach to modeling triadic comparison data. The complete and coherent handling of uncertainty offered by the probabilistic approach using Bayesian methods is particularly important, given evidence that traditional MDS methods-non-Bayesian methods that do not represent uncertainty - can be very sensitive to small changes in triadic comparison data (Voorspoels et al., 2014). To understand the semantic structures inferred from triadic comparisons, it is important to know not just what the most likely representation is, but how much confidence can be

\footnotetext{
${ }^{1}$ JAGS code is provided in the Appendix.
} 
placed in that representation. Bayesian inference represents and conveys this uncertainty.

\section{Results}

We applied the graphical model in Fig. 5 independently to the first 277 subjects in each of groups I-VIII. We equated the number in each group to assist in the analysis of the results in ways we describe below. The total of 277 was chosen because it was the number of subjects in the smallest group, as detailed in Fig. 1. We assume throughout this paper that representations are two-dimensional and use the Euclidean distance metric, consistent with many previous analyses. The graphical modeling framework obviously allows for more general representational spaces and metrics, and we return to this issue in the discussion. Our results are based on three independent chains each containing 2000 samples collected after 5000 discarded burn-in samples. The chains were checked visually for convergence, as well as by the standard $\hat{R}$ statistic (Brooks \& Gelman, 1997), and a single chain was then used for subsequent analysis. Every sample in this chain was post-processed to remove the lack of invariance coming from translation, reflection, and rotation in a two-dimensional Euclidean MDS representation (Bakker \& Poole, 2013, p. 130).

Figure 6 shows the representations inferred for each of groups I-VIII. The posterior mean for the location for each animal is shown as a black circle, and is labeled. All of the posterior samples from the retained chain are shown in gray, and indicate the uncertainties about the spatial locations. This uncertainty is a measure of the confidence in the representation of each animal that is justified by the triadic comparison data. Because this analysis uses an equal number of subjects in each severity group, the uncertainty in the stimulus locations shown by the gray points can be interpreted as the uncertainty that group of subjects has about the representation of the animal stimuli.

The successive degradation in the semantic representation of the animals with the severity of impairment in group I through group VIII is clearly evident. There is an increase in uncertainty about the locations of the stimuli. Accompanying the increase in uncertainty, the MDS representations in Fig. 6 show a loss of the clustering of semantically related animals that is evident for group I. The natural groupings of semantically related animals - such as giraffe and elephant, or lion and tiger, or chimpanzee, monkey, and gorilla, or rat, rabbit, chipmunk, and beaver-is progressively lost in the representations of more impaired groups. Interestingly, some of these clusters are lost earlier than others with, for example, the chimpanzee, monkey, and gorilla cluster still evident in the representations of group VI.

\section{Measure of semantic clustering}

While the changes in semantic clustering in Fig. 6 are visually apparent, it is still desirable to have a measure that is able to quantify these changes. We investigated the ability of a measure of spatial randomness, based on nearest neighbor distances, to detect the changes in semantic clustering. This measure comes from the field of spatial statistics (e.g., Ripley, 1981), and has been used in the study of human perceptual processing (Dry et al., 2012). The spatial randomness measure-derived independently, apparently, by Hertz (1909) and Clark and Evans (1954) - is the ratio of observed mean nearest neighbor distance for a configuration of points to the mean nearest neighbor distance that would be expected if the points were completely spatially randomly configured. Formally, the measure is $R=r_{\mathrm{O}} / r_{\mathrm{E}}$, where $r_{\mathrm{O}}=\frac{1}{n} \sum_{i \neq j} \min \left\{d_{i j}\right\}$ is the observed mean nearest neighbor distance, and the expected mean $r_{\mathrm{E}}$ was calculated in the same way based on the distances between points across a large number of random $n$-point configurations. Both the observed and random configurations were scaled to lie in the unit square, and the random configurations were generated by choosing the two coordinate locations independently and uniformly in the unit interval.

Figure 7 provides examples of the spatial randomness measure that provide the intuition for the way in which it measures clustering structure. The panels on the righthand side, following the examples provided by Dry et al. (2012), show nine different configurations of points, and their associated $R$ measures. Complete spatial randomness corresponds to $R=1$, when the observed and expected mean nearest neighbor distances are the same. Values of $R$ smaller than one occur as the nearest neighbor distances in the configuration of points decrease, which naturally occurs when the points cluster. The more extreme this clustering, the smaller the $R$ measure. Values of $R$ larger than one occur as the nearest neighbor distances in the configuration of points increase, which naturally occur as points form a regular grid. The more regular this arrangement, the larger the $R$ measure.

The main panel on the left-hand side of Fig. 7 applies the spatial randomness measure to the MDS representations in Fig. 6. A distribution of the $R$ measure is naturally obtained, by calculating the $R$ value for every configuration in the posterior distribution of the MDS representations. These distributions can be considered as posterior distributions for $R$, expressing the uncertainty about $R$ for each of the eight groups. It can be seen that, while there is considerable uncertainty leading to overlap in the posterior distributions, there is a general increase in the $R$ measure as groups become more severely impaired. For less impaired groups the $R$ measure is less than 1, indicating 


\section{Group I}

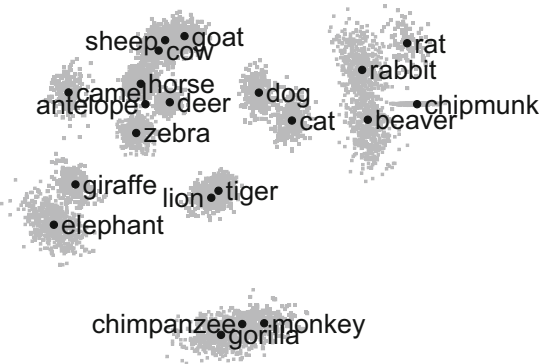

Group III

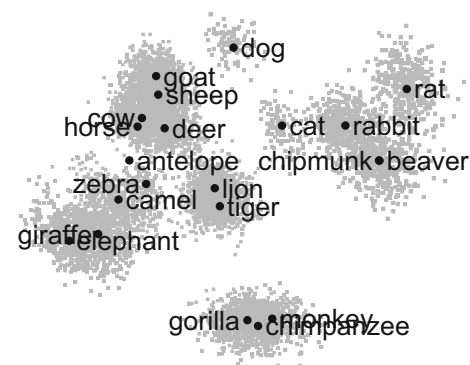

\section{Group V}

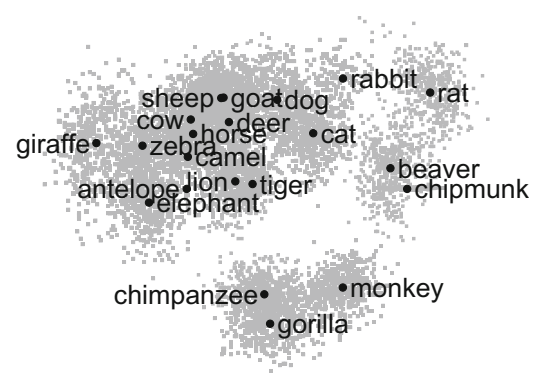

\section{Group VII}

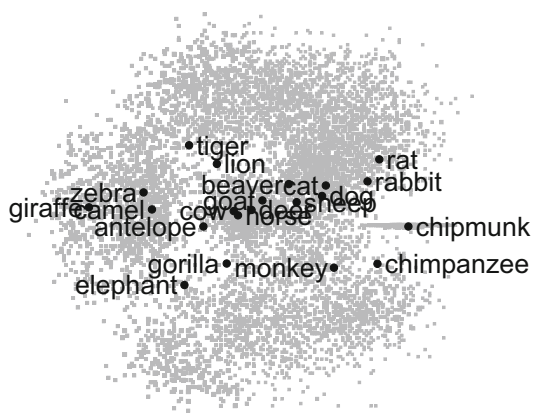

\section{Group II}

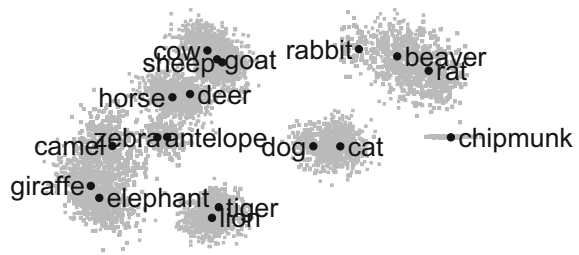

gqfilarey chimpanzee
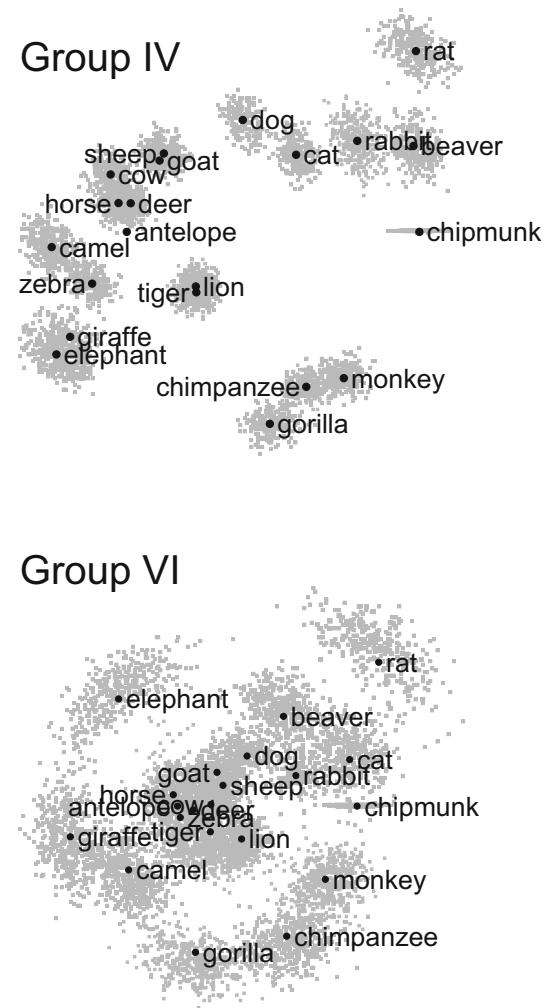

Group VIII

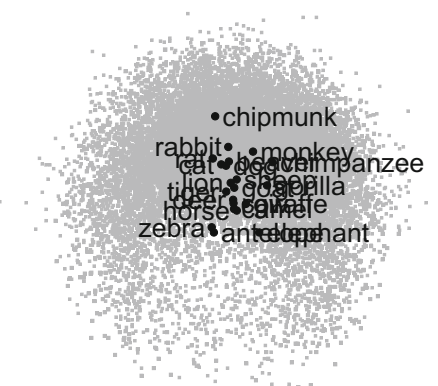

Fig. 6 Inferred two-dimensional multidimensional scaling representations of the animals, based on the generative model of triadic comparisons, for groups I-VIII

the presence of cluster structure. For more impaired groups, the $R$ measure increases towards 1 , indicating a spatially random MDS configuration, lacking in semantic clustering. In this way, the $R$ measure provides a quantification 
of the degree of cluster structure visually evident in Fig. 6.

\section{Individual multidimensional scaling model}

The group MDS model examined differences in the semantic representation of pre-defined groups of people. A complementary analysis builds assumptions about individual differences into the MDS model. As discussed earlier, previous research has focused on well-established extensions of MDS to individual differences, such as the INDSCAL model that assumes individuals give different weights to different stimulus dimensions. We pursue a different approach to formalizing individual differences, involving how deterministically the odd one out choices a person makes follow the inferred underlying semantic representation of the stimuli. The basic intuition is that this determinism provides a measure of the severity of impairment, with less impaired people closely following the choices predicted by the semantic structure, and more impaired people deviating from those predictions. Thus, this model takes the theoretical perspective that memory impairment can be understood in terms of impaired access to a common semantic representation.

\section{Generative model}

To incorporate individual differences, we make the key assumptions that all of the subjects rely on the same MDS representation of the animals, but that memory impairment affects their ability to make triadic comparison choices consistent with this representation. The universality of the

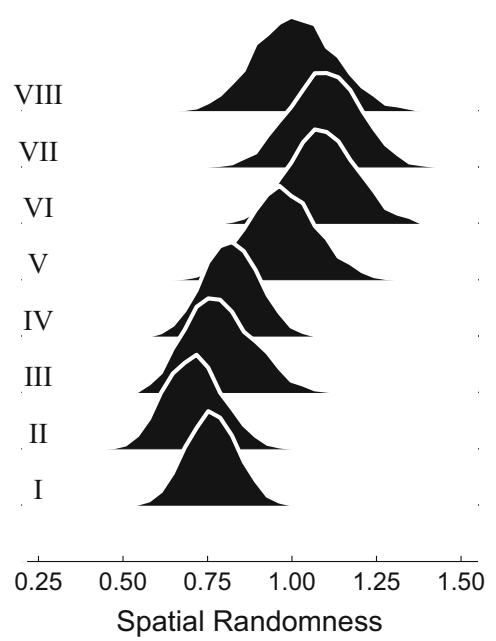

Fig. 7 Spatial randomness measure analysis of the MDS solutions. The left-hand panel shows the posterior distribution over the spatial randomness measure $R$ for groups I-VIII. The right-hand panels semantic representation of animal terms has a theoretical and empirical justification from a body of work in cultural anthropology that models culture as shared cognitive representations, and presents evidence for the constancy of MDS representations of animals, as well as color names and kinship terms (Romey and Moore, 1998). The individual differences in how this shared latent semantic representation generates decisions are modeled using a version of the Luce-choice rule (Luce, 1959), in which the similarities between stimulus representations are affected by an individual-level response determinism parameter, as previously developed in models of category learning (e.g., Ashby \& Maddox, 1993; McKinley \& Nosofsky, 1995). Formally, the probability that the first stimulus will be chosen as the odd one out is based on the exponentiated similarity of the other two stimuli, giving the choice probability $\theta_{1}=$ $\frac{1}{Z} \exp \left(-d_{23}\right)^{\gamma}=\frac{1}{Z} \exp \left(-\gamma d_{23}\right)$, where $\gamma$ is the positive response determinism parameter, and $Z$ is again a normalizing constant insuring the three choice probabilities sum to one.

Intuitively, the value of $\gamma$ controls how deterministically the responses based on the choice probabilities follow the underlying similarities. When $\gamma=1$, the Luce-choice rule reduces to the choice probabilities used in the graphical model in Fig. 5. This corresponds to probability matching so that, for example, if the three pairwise similarities are $0.6,0.3$, and 0.1 then $\theta=(0.6,0.3,0.1)$, and the first stimulus will be chosen $60 \%$ of the time, the second $30 \%$, and the third $10 \%$. As $\gamma$ increases above 1 , the largest similarity 0.6 will generate a relatively larger choice probability. For example, with $\gamma=2$ the choice probabilities become $\theta \approx(0.885,0.111,0.004)$, so that the first stimulus is now more likely to be chosen. In other words, as

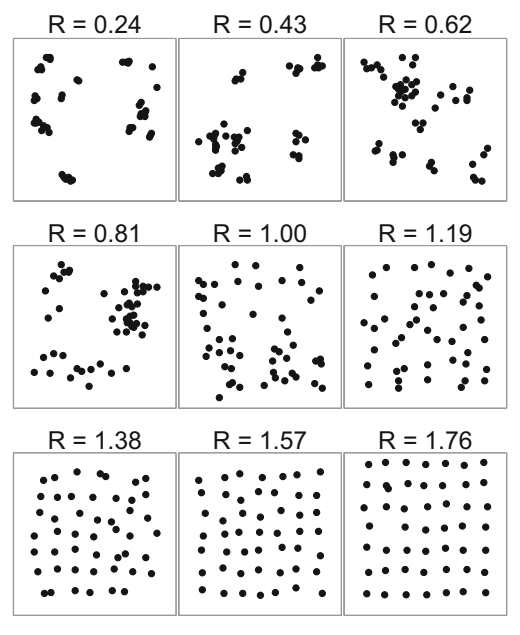

show examples of the $R$ measure for a range of constructed point configurations (based on Dry et al., 2012, Figure 2) 


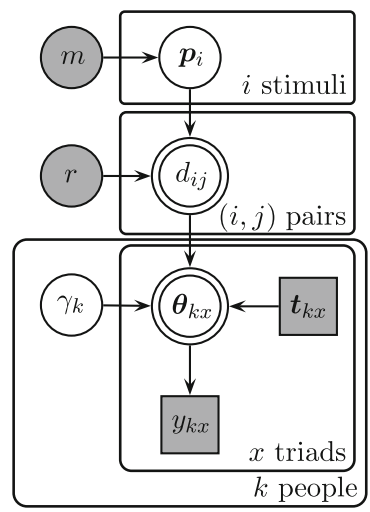

$$
\begin{aligned}
\boldsymbol{p}_{i} & \sim \operatorname{Uniform}(-10,10)^{m} \\
d_{i j} & \leftarrow\left(\sum_{m^{\prime}}\left|p_{i m^{\prime}}-p_{j m^{\prime}}\right|^{r}\right)^{1 / r} \\
\gamma_{k} & \sim \operatorname{Gamma}(2,1) \\
\boldsymbol{\theta}_{k x} & \leftarrow{ }_{Z}^{1}\left(\exp \left(-\gamma_{k} d_{t_{k x 2}, t_{k x 3}}\right), \exp \left(-\gamma_{k} d_{t_{k x 1}, t_{k x 3}}\right), \exp \left(-\gamma_{k} d_{t_{k x 1}, t_{k x 2}}\right)\right) \\
y_{k x} & \sim \text { Categorical }\left(\boldsymbol{\theta}_{k x}\right)
\end{aligned}
$$

Fig. 8 Graphical model for inferring a spatial representation based on triadic comparisons, incorporating individual-level differences in the determinism of comparisons

the determinism parameter $\gamma$ increases, the stimulus that is least similar to the others according to the MDS representation becomes chosen with increasingly higher probability. In contrast, as $\gamma$ decreases below 1 towards 0 , the probabilities move towards $\theta=\left(\frac{1}{3}, \frac{1}{3}, \frac{1}{3}\right)$ regardless of the underlying similarities, and each stimulus becomes equally likely to be chosen. In this way, the determinism parameter spans a range from chance responding as $\gamma \rightarrow 0$, to probability matching at $\gamma=1$, to deterministic responding as $\gamma \rightarrow \infty$, and can be interpreted as measuring how closely an individual's choices adhere to an underlying MDS representation.

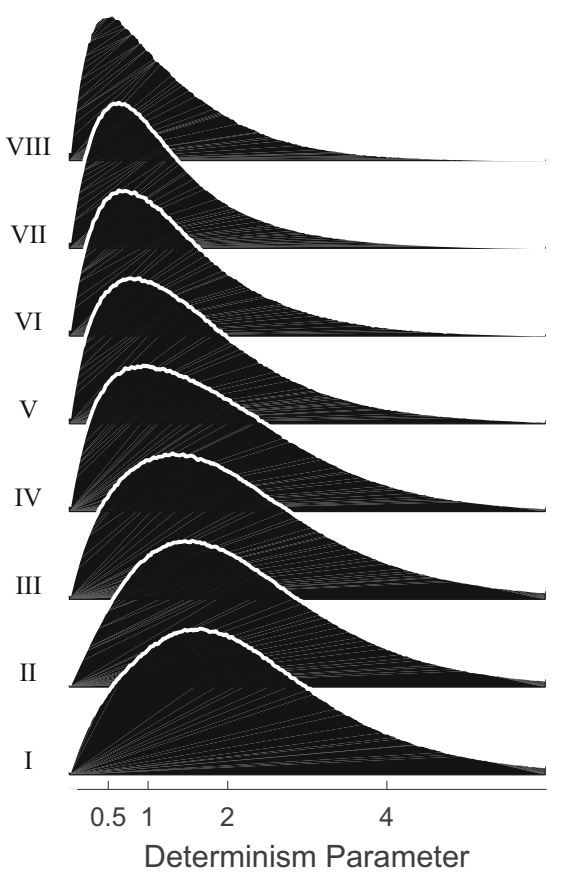

Fig. 9 Inferred determinism parameter values for groups and individuals. The left-hand panel shows the posterior uncertainty over $\gamma$ for groups I-VIII. The right-hand panels show the distribution (in black)
The graphical model for the individual-level analysis, using the Luce-choice rule approach, is shown in Fig. 8. The MDS representation still has the $i$ th stimuli represented by the coordinate location $p_{i}$ in an $m$-dimensional space, generating pairwise distances $d_{i j}$ under the Minkowski $r$ metric. For the $k$ th person, these distances generate choice probabilities for the $x$ th triad, $\theta_{k x}$ according to their determinism, $\gamma_{k}$, which is given a $\operatorname{Gamma}(2,1)$ prior. This prior was chosen so that the mode is at the special case $\gamma=1$ of probability matching, but larger and smaller values are given significant prior density. The behavioral data are again categorically distributed according to the
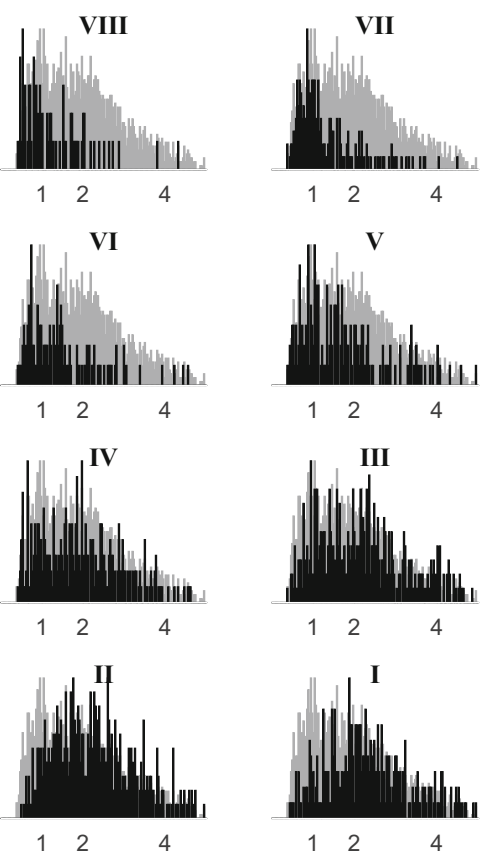

of the posterior expectation of $\gamma$ for each individual in groups I-VIII, referenced against the distribution (in gray) for all individuals in all groups 
choice probabilities. Setting a theoretically informed prior is important for $\gamma$, because the model's likelihood function has in an inherent indeterminacy, whereby a scalar change in distances between points can be counteracted by reducing $\gamma$ by the same scale factor. The informative prior expresses a theoretical expectation about the likelihood of probability matching and other sorts of behavior-as outlined above-and provides a scale for $\gamma$ that breaks this indeterminacy.

\section{Results}

We applied the graphical model in Fig. 8 to the first visit data for all of the 2,162 subjects. Our results are based on three independent chains each containing 5000 samples collected after 5000 discarded burn-in samples. The chains were again checked visually and with the $\hat{R}$ statistic for convergence. Figure 9 presents the main results, focusing on the determinism parameter $\gamma$. The left-hand panel shows the posterior distribution over the $\gamma$ parameter for all those subjects in each severity group. That is, it combines the posterior samples for every subject in each group, to form an overall distribution. The group membership information is not available to the model, so this analysis is best interpreted as testing whether the determinism parameter approach to individual differences is able to measure differences in the severity of impairment of the groups. While the posterior distributions in the left-hand panel of Fig. 9 overlap significantly, they do progress from larger to smaller values as severity increases from group I to group VIII, corresponding to more deterministic responding, for the least impaired groups, and more random responding, for the more impaired groups.

The smaller panels on the right-hand side of Fig. 9 present a more applied analysis of the individual differences model. Each panel corresponds to the subjects in one of the severity groups, showing the distribution of the posterior expectation of their $\gamma$ parameter. This means that, in these analyses, each person's response determinism is summarized by a point estimate, and the distribution of these point estimates over all the subjects in the group is shown in black. In all of the panels, the entire distribution of these point summaries for all subjects is shown in gray, to allow visual comparison. It is clear that the distributions of these individual-level measures shift from larger to smaller values as severity increases. The posterior expectations of the determinism parameter $\gamma$ do not clearly separate individual subjects into different severity groups, but instead show considerable overlap. This is not surprising, since the analysis at an individual level is based on just 12 triadic comparison choices involving only nine of the 21 animal stimuli. It is possible the information latent in these data is not sufficient for confident and accurate measures of a subject's severity of impairment under any analysis. What the current results show-through the clear trends in the individual estimates in Fig. 9-is that the approach to modeling individual differences developed here is potentially useful, because it captures useful information about severity at the level of specific subjects on specific testing occasions.

\section{Discussion}

The goal of this paper is to develop and demonstrate new methods, measures, and models for understanding changes in semantic representation, and access to semantic representation, with memory impairment. We focused on MDS representations of semantic structure, and developed a simple cognitive process model to relate these representations to choosing the odd one out in a triadic comparison task. From a theoretical perspective emphasizing the possible effect of impairment on semantic representation, we inferred MDS representations for different subject groups. The results in Fig. 6 show clear and interpretable changes in semantic representation across the groups of subjects with different levels of memory impairment. In particular, there is a progressive breakdown in semantic structure, with the cluster relationships between similar stimuli being degraded. We proposed that important aspects of this change in cluster structure could be measured by a previously developed statistic that measures spatial randomness. Applying the statistic to the MDS representations produced interpretable results, with less impaired subject groups showing semantic cluster organization, and more impaired groups showing near-random organization. The results in Fig. 6 also demonstrate the usefulness of the Bayesian methods we use, coming from their basis in representing uncertainty. The representations for the different groups characterize not just the most likely representation of the animals, but show how much certainty or confidence can be placed in different possible representations. This uncertainty information is useful because it captures an important aspect of the degradation in semantic structure with impairment.

From the theoretical perspective emphasizing changes in access to semantic representation with impairment, we developed a new approach to incorporating individual differences. Previous individual differences models for MDS representations, especially involving the INDSCAL model, have often assumed that individual differences can be understood in terms of different people attending to the dimensions of the stimuli in different ways. This sort of selective attention mechanism is successfully and intuitively used to model cognitive phenomena like category learning (e.g., Kruschke, 1992; Nosofsky, 1984, 1986), but seems 
most applicable when there are clear and separable underlying dimensions that characterize the stimuli. That is not the case for the MDS representations we consider, since they are only two-dimensional and rely on the Euclidean distance metric which theoretically corresponds to integral rather than separable stimuli (Garner, 1974; Shepard, 1987). Rather than consider higher-dimensional representations with a city-block metric-which would, in principle, be possible in our Bayesian framework by changing $m$ and $r$ in the graphical models-we focused on a different approach to individual differences. The basic assumption of the new approach is to assume there is shared MDS representation of the animal names, but different people make odd one out decisions that are consistent with this representation to different degrees. Formalizing these assumptions led to a new model in which individual differences are parameterized in terms of response determinism, which can be conceived as a measure of how consistently an individual makes the choices predicted by the underlying representation. The application of this model to our data showed that, even though each individual makes a very small number of decisions considering only a subset of the stimuli, the determinism parameter permits useful inferences about memory impairment at the level of individuals.

Overall, our use of two model based on MDS representations and triadic choice decisions-one model focusing on differences in representations, and the other focusing on differences in decisions-identified relationships between semantic clustering, response determinism, and memory impairment that was not evident from standard analyses of the same data. Given that some of these data are from a clinical practice, and that the 12 triads assessed per subject was small enough to be doable even in primary care settings, the results of both models are impressive, and support the potential for model-based clinical data analysis (Bayles et al., 1990; Chan et al., 1995; Chan et al., 2001; Silveri et al., 1991; Tallent et al., 2001; van Ravenzwaaij et al., 2011; Wetzels et al., 2010). Further testing of the relationship between semantic organization and memory impairment could be achieved by considering independent assessments of the severity of impairment. As mentioned earlier, one obvious possibility is the FAST staging measure of functional impairment (Reisberg, 1988). Diagnoses with relatively early frontal pathology and executive dysfunction, such as multiple sclerosis, Parkinson's disease, Frontal Temporal Lobe disease, and cerebrovascular disease, could be evaluated by applying the models to very mildly impaired subjects and comparing results to those of very mildly impaired subjects with relatively early medial temporal pathology, such as epilepsy, anoxia, hippocampal sclerosis, and Alzheimer's disease. Further refinements of the models may be possible by using different underlying assumptions including different representational models (Borg \& Lingoes, 1987; Navarro \& Lee, 2003; 2004; Shepard, 1980), and cognitive processes for relating stimulus similarity to choice behavior (Goldstone, 1999; Luce, 1959; Restle, 1961; Thurstone, 1927). Given the demonstrated promise of the models, measures, and methods we have developed, and the rich possibilities for further investigation, we hope joint reliance on cognitive models and Bayesian methods will become a standard approach in study of the effect of memory impairment on semantic representation.

Acknowledgments We thank Matt Dry and James Pooley for helpful discussions, and Rob Nosofsky, Gert Storms, and an anonymous reviewer for feedback on an earlier version of this paper. We also thank Dr. Douglas Trenkle for providing data from his primary care practice in Maine, and Mr. Marc Cohen and his team at Life Plans for providing the long-term care insurance sample data.

\section{Appendix}

The JAGS script implementing the graphical model in Fig. 5 is shown below. The inputs are the data nStim giving the total number of stimuli, nTrial giving the total number of triadic comparison trials, $t[i, 1: 3]$ giving the indices to the three stimuli presented on the $i$ th trial, and $\mathrm{y}$ [i] indicating whether the first, second, or third stimulus was chosen on the $i$ th trial.

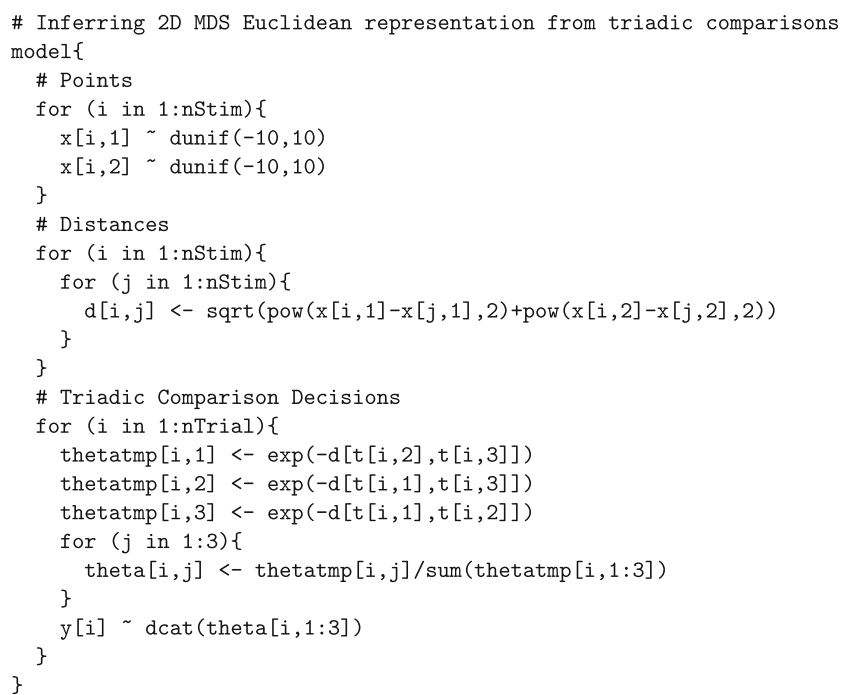

The JAGS script implementing the graphical model in Fig. 8 is shown below. It extends the earlier graphical model by introducing an individual-level response determinism parameter gamma [k] for the $k$ th individual, which requires as additional input $\mathrm{p}[\mathrm{i}]$ indicating which individual is associated with the $i$ th trial. 


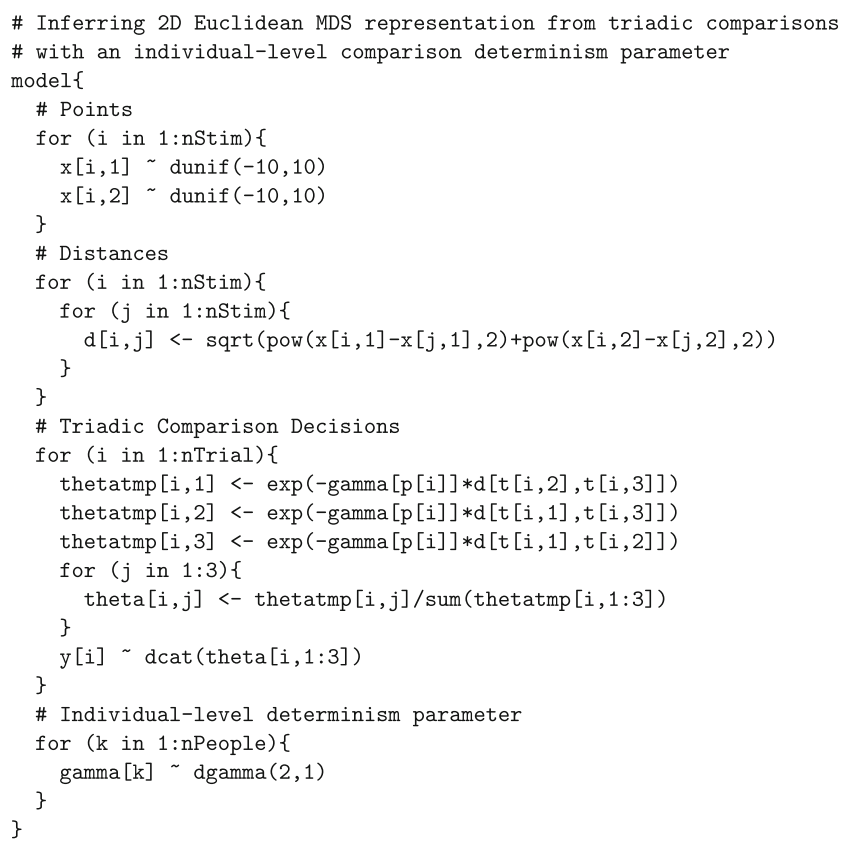

\section{References}

Arabie, P., Carroll, J. D., \& DeSarbo, W. S. (1987). Three-way scaling and clustering. Newbury Park: Sage.

Ashby, F.G., \& Maddox, W. T. (1993). Relations between prototype, exemplar, and decision bound models of categorization. Journal of Mathematical Psychology, 37, 372-400.

Au, A., Chan, A.S., \& Chiu, H. (2003). Conceptual organization in Alzheimer's dementia. Journal of Clinical and Experimental Neuropsychology, 25, 737-750.

Bakker, R., \& Poole, K.T. (2013). Bayesian metric multidimensional scaling. Political Analysis, 21, 125-140.

Bayles, K.A., Tomoeda, C.K., \& Trosset, M.W. (1990). Naming and categorical knowledge in Alzheimer's disease: the process of semantic memory deterioration. Brain and Language, 39, 498510

Borg, I., \& Lingoes, J. (1987). Multidimensional similarity structure analysis. New York: Springer.

Bousfield, W.A. (1953). The occurrence of clustering in the recall of randomly arranged associates. The Journal of General Psychology, 49, 229-240.

Brooks, S.P., \& Gelman, A. (1997). General methods for monitoring convergence of iterative simulations. Journal of Computational and Graphical Statistics, 7, 434-455.

Burton, M.L., \& Nerlove, S.B. (1976). Balanced designs for triads tests: two examples from English. Social Science Research, 5, 247-267.

Chan, A.S., Butters, N., Salmon, D.P., Johnson, S.A., Paulsen, J.S., \& Swenson, M.R. (1995). Comparison of the semantic networks in patients with dementia and amnesia. Neuropsychology, 9, 177.

Chan, A.S., Salmon, D.P., \& De La Pena, J. (2001). Abnormal semantic network for "animals" but not "tools" in patients with Alzheimer's disease. Cortex, 37, 197-217.

Clark, P.J., \& Evans, F.C. (1954). Distance to nearest neighbor as a measure of spatial relationships in populations. Ecology, 35, 445453.
Corter, J. E. (1996). Tree models of similarity and association. Thousand Oaks: Sage.

Dry, M.D., Preiss, A.K., \& Wagemans, J. (2012). Clustering, randomness, and regularity: spatial distributions and human performance on the traveling salesperson problem and minimum spanning tree problem. Journal of Problem Solving, $4,1-17$.

Elvevåg, B., \& Storms, G. (2003). Scaling and clustering in the study of semantic disruptions in patients with schizophrenia: a re-evaluation. Schizophrenia Research, 63, 237-246.

Elvevåg, B., Fisher, J., Gurd, J., \& Goldberg, T. (2002). Semantic clustering in verbal fluency: schizophrenic patients versus control participants. Psychological Medicine, 32, 909-917.

Elvevåg, B., Heit, E., Storms, G., \& Goldberg, T. (2005). Category content and structure in schizophrenia: an evaluation using the instantiation principle. Neuropsychology, 19, 371

Fagundo, A.B., López, S., Romero, M., Guarch, J., Marcos, T., \& Salamero, M. (2008). Clustering and switching in semantic fluency: predictors of the development of Alzheimer's disease. International Journal of Geriatric Psychiatry, 23, 1007-1013.

Garner, W. R. (1974). The processing of information and structure. Erlbaum: Potomac.

Goldstone, R. L. (1999). Similarity. In Wilson, R.A., \& Keil, F.C. (Eds.) MIT Encyclopedia of the Cognitive Sciences (pp. 763765). Cambridge: MIT Press.

Gordon, A. D. (1999). Classification, Second Ed. London: Chapman $\&$ Hall/CRC Press.

Hertz, P. (1909). Uber den geigenseitigen durchschnittlichen abstand von punkten, die mit bekannter mittlerer dichte im raume angeordnet sind. Mathematische Annalen, 67, 387-398.

Kruschke, J.K. (1992). ALCOVE: an exemplar-based connectionist model of category learning. Psychological Review, 99, 22-44.

Lee, M.D. (2011). How cognitive modeling can benefit from hierarchical Bayesian models. Journal of Mathematical Psychology, 55, $1-7$.

Lee, M. D., \& Wagenmakers, E. J. (2013). Bayesian cognitive modeling: a practical course: Cambridge University Press.

Luce, R. D. (1959). Detection and recognition. In Luce, R. D., Bush, R. R., \& Galanter, E. (Eds.) Handbook of mathematical psychology (pp. 103-189). New York: Wiley.

McKinley, S.C., \& Nosofsky, R.M. (1995). Investigations of exemplar and decision bound models in large, ill-defined category structures. Journal of Experimental Psychology: Human Perception and Performance, 21, 128.

Navarro, D. J., \& Lee, M. D. (2003). Combining dimensions and features in similarity-based representations. In Becker, S., Thrun, S., \& Obermayer, K. (Eds.) Advances in Neural Information Processing Systems, (Vol. 15 pp. 59-66). Cambridge, MA: MIT Press.

Navarro, D.J., \& Lee, M.D. (2004). Common and distinctive features in stimulus similarity: A modified version of the contrast model. Psychonomic Bulletin \& Review, 11, 961-974.

Nosofsky, R. M. (1984). Choice, similarity, and the context theory of classification. Journal of Experimental Psychology: Learning, Memory, and Cognition, 10(1), 104-114.

Nosofsky, R.M. (1986). Attention, similarity and the identificationcategorization relationship. Journal of Experimental Psychology: General, 115, 39-57.

Nosofsky, R.M. (1992). Similarity scaling and cognitive process models. Annual Review of Psychology, 43, 25-53.

Okada, K., \& Shigemasu, K. (2009). BMDS: A collection of R functions for Bayesian multidimensional scaling. Applied Psychological Measurement, 33, 570-571. 
Plummer, M. (2003). JAGS: A program for analysis of Bayesian graphical models using Gibbs sampling. In Hornik, K., Leisch, F., \& Zeileis, A. (Eds.) Proceedings of the 3rd International Workshop on Distributed Statistical Computing. Austria: Vienna.

Reisberg, B. (1988). Functional assessment staging (FAST). Psychopharmacology Bulletin, 24, 653-659.

Restle, F. (1961). Psychology of judgment and choice. New York: Wiley.

Ripley, B. D. (1981). Spatial statistics: Wiley.

Romey, A.K., \& Moore, C.C. (1998). Toward a theory of culture as shared cognitive structures. Ethos, 26, 314-337.

Romney, A.K., Brewer, D.D., \& Batchelder, W.H. (1993). Predicting clustering from semantic structure. Psychological Science, 4, 28 34.

Schvaneveldt, R.W., Durso, F.T., \& Dearholt, D.W. (1989). Network structures in proximity data. Psychology of Learning and Motivation, 24, 249-284.

Shepard, R.N. (1974). Representation of structure in similarity data: problems and prospects. Psychometrika, 39, 373-422.

Shepard, R.N. (1980). Multidimensional scaling, tree-fitting, and clustering. Science, 214, 390-398.

Shepard, R.N. (1987). Toward a universal law of generalization for psychological science. Science, 237, 1317-1323.

Silveri, M.C., Daniele, A., Giustolisi, L., \& Gainotti, G. (1991). Dissociation between knowledge of living and nonliving things in dementia of the Alzheimer type. Neurology, 41, 545-545.
Storms, G., Dirikx, T., Saerens, J., Verstraeten, S., \& De Deyn, P.P. (2003). On the use of scaling and clustering in the study of semantic deficits. Neuropsychology, 17, 289.

Sylvester, C. Y.C., \& Shimamura, A.P. (2002). Evidence for intact semantic representations in patients with frontal lobe lesions. Neuropsychology, 16, 197.

Tallent, K.A., Weinberger, D.R., \& Goldberg, T.E. (2001). Associating semantic space abnormalities with formal thought disorder in schizophrenia: use of triadic comparisons. Journal of Clinical and Experimental Neuropsychology, 23, 285296.

Thurstone, L.L. (1927). A law of comparative judgement. Psychological Review, 34, 273-286.

van Ravenzwaaij, D., Dutilh, G., \& Wagenmakers, E. J. (2011). Cognitive model decomposition of the BART: assessment and application. Journal of Mathematical Psychology, $55,94-105$.

Voorspoels, W., Storms, G., Longenecker, J., Verheyen, S., Weinberger, D.R., \& Elvevåg, B. (2014). Deriving semantic structure from category fluency: clustering techniques and their pitfalls. Cortex, 55, 130-147.

Wetzels, R., Vandekerckhove, J., Tuerlinckx, F., \& Wagenmakers, E. J. (2010). Bayesian parameter estimation in the Expectancy Valence model of the Iowa gambling task. Journal of Mathematical Psychology, 54, 14-27.

Zinnes, J.L., \& MacKay, D.B. (1983). Probabilistic multidimensional scaling: Complete and incomplete data. Psychometrika, 48, 27-48. 